\title{
Involvement of chromatin and histone acetylation in the regula- tion of HIV-LTR by thyroid hormone receptor
}

\author{
Shao Chung Victor HSIA, Hua WANG, Yun Bo SHI* \\ Unit on Molecular Morphogenesis, Laboratory of Molecular Embryology, National Institute of Child Health \\ and Human Development, National Institute of Health, Building 18T, Room 106, Bethesda, MD 20892-5431, \\ $U S A$
}

\begin{abstract}
The HIV-1 LTR controls the expression of HIV-1 viral genes and thus is critical for viral propagation and pathology. Numerous host factors have been shown to participate in the regulation of the LTR promoter. Among them is the thyroid hormone $\left(\mathrm{T}_{3}\right)$ receptor (TR). TR has been shown to bind to the critical region of the promoter that contain the NF к B and Sp1 binding sites. Interestingly, earlier transient transfection studies in tissue culture cells have yielded contradicting conclusions on the role of TR in LTR regulation, likely due to the use of different cell types and/or lack of proper chromatin organization. Here, using the frog oocyte as a model system that allows replication-coupled chromatin assembly, mimicking that in somatic cells, we demonstrate that unliganded heterodimers of TR and RXR (9-cis retinoic acid receptor) repress LTR while the addition of T3 relieves the repression and further activates the promoter. More importantly, we show that chromatin and unliganded TR/RXR synergize to repress the promoter in a histone deacetylase-dependent manner.
\end{abstract}

Key words: HIV, thyroid hormone receptor, histone acetylation, transcriptional repression, Xenopus oocyte.

\section{INTRODUCTION}

Proviral gene expression is essential for the propagation and pathology of the human immunodeficiency virus (HIV) type 1 (HIV-1). The long terminal repeat (LTR) of HIV directs the transcription of the proviral message. Numerous studies have been carried out to determine the sequence requirement of the LTR and to identify transacting factors. These earlier studies have shown that the LTR promoter is tightly regulated by multiple viral and host proteins through specific DNA elements within the LTR[1]. The DNA elements important for LTR

*Abbreviations: $\mathrm{T}_{3}$, Thyroid Hormone; TR, Thyroid Hormone Receptor; HAT, histone acetyl transferase; HDAC, histone deacetylase; TSA, Trichostatin A; HIV: human immunodeficiency virus; LTR: Long terminal repeat

*Corresponding Author: Dr. Yun-Bo SHI, Tel: (301) 402-1004, Fax: (301) 402-1323, E-mail: shi@helix.nih.gov

Received Aug-28-2000 Revised Oct-19-2000 Accepted Oct-20-2000 activity are located within the region from -454 to +184 , where +1 is the transcription start site. Of particular interest is the region from -104 to +1 , where the TATA box and the essential DNA binding sites for the transcription factors $\mathrm{Sp} 1$ and $\mathrm{NF} \kappa \mathrm{B}$ are present (Fig 1)[1],[2].

The involvement of multiple host factors in HIV1 transcription suggests that HIV-1 propagation and the pathological progression of AIDS and AIDS-related complex (ARC) are likely to vary depending upon the physiological conditions that can regulate the availability and function of these host factors. Thyroid hormone $\left(\mathrm{T}_{3}\right)$ and its receptors (TRs) have been suggested to play a role in HIV development. First, AIDS or ARC patients have multiple endocrine abnormalities and their serum T3 levels are often altered[3], [4]. Low levels of T3 
often correlate with survival rate, and in all levels of illness, there is an increase in T3-binding globulin levels. Thus, modulation of cellular T3 concentrations may affect HIV pathological progression. Furthermore, TRs are expressed in lymphocytes and other HIV-1 target tissues[5], [6]. More importantly, several reports[7-9] have identified T3 response elements (TREs) within the LTR that can be recognized by TR/RXR (9-cis-retinoic acid receptor) heterodimers, which mediate the effects of T3[10-12]. Cotransfection of promoters bearing these sites and a vector over-expressing a TR results in T3-dependent transcriptional regulation. Surprisingly, these studies yielded contradicting conclusions as TR was found to activate HIV LTR in the absence of T3 but not in the presence in one [8] while the opposite was reported in the other two[7], [9]. One possible reason for this controversy is due to the use of different cell lines in the studies.

TRs are ligand (T3)-dependent transcription factors belonging to the superfamily nuclear hormone receptors. They bind to TREs present in their target genes and activate or repress transcription in the presence or absence of T3, respectively[10-12]. While TRs can bind to TREs as monomers or homodimers, in vivo, they most likely function as heterodimers formed with RXRs[10-15]. TRs, similar to at least some other members of this family such as glucocorticoid receptor, can bind to their DNA targets in chromatin and cause chromatin remodeling in the presence of T3[16-18]. Furthermore, chromatin appears to also play an important role in transcriptional repression by unliganded TRs[16], [17]. Thus, another possibility for the contradicting findings on the role of TR in HIV LTR regulation is that the earlier studies were done in tissue culture cells by transient transfections. Under such conditions, the promoter templates are in a chromatin state distinct from that in somatic cells.

Thus, to properly study the regulation of HIV-1 LTR by T3, one needs an in vivo, system where the promoter can be analyzed in its native chromosomal environment. Here, we have made use of a unique property of Xenopus laevis oocytes. That is, when single-stranded (ss) DNA is injected into oocyte nucleus, it is quickly (within $2 \mathrm{~h}$ ) replicated and simultaneously chromatinized in a replication- coupled chromatin assembly pathway, resembling that in normal somatic cells[19]. In addition, the oocyte stores large quantities of basal machinery for translation, replication, and transcription, but is lacking or limited in many transcription factors such as TRs and RXRs[20], making it possible to investigate the function of these factors. Furthermore, the high degree of conservation of the transcriptional machinery and other factors suggest that the results from this model system will be equally applicable to human.

Using this system, we demonstrate that TR/RXR can repress LTR in the context of chromatin. While different chromatin assembly conditions have little effect on the basal LTR activity, replication-coupled chromatin assembly synergizes with unliganded TR/RXR to repress LTR. Furthermore, the addition of either T3 or trichostatin A (TSA), a specific inhibitor of histone deacetylases[21], relieves this repression, suggesting that transcriptional regulation by TR/RXR of chromatinized LTR involves histone acetylation.

\section{MATERIALS AND METHODS}

\section{Plasmids}

The plasmid $\mathrm{p} 4 / 5 \mathrm{~A}$ containing the core HIV-1 LTR promoter was derived from the $\mathrm{pTRbA}$ construct[16] in which the TR $\beta \mathrm{A}$ promoter was replaced by a $131 \mathrm{bp}$ PCR-generated fragment from HIV-1 5' LTR (Fig 1). Forward primer LTR4 (5' GGGGTACCACAAGGGACTTTCCCT-3' ) and backward primer LTR5 (5' -GAAGATCTAGGCTTAAGCAGTGGG-3' ) containing restriction sites $\mathrm{KpnI}$ and BglII, respectively, were used for PCR amplification of the LTR region from -108 to +72 . The double digested product $(-108$ to +19 , due to an internal BglII site at +19 ) was ligated into the KpnI-BglII linearized pTR $\beta \mathrm{A}$ to produce the $\mathrm{p} 4 / 5 \mathrm{~A}$, which contained $300 \mathrm{bp}$ of the $5^{\prime}$ portion of the CAT gene downstream of the LTR and phage f1 replication origin in the pBluescript KS (-) vector (Stratagene). Both single- (ss) and double-stranded (ds) p4/5A DNA were generated according to Maniatis et al.[22].

\section{In vitro synthesis of capped RNA}

The in vitro transcription was performed using the mMESSAGE mMACHING ${ }^{T M}$ SP6 kit (Ambion, TX) essentially as described by the manufacturer. The templates used for the reactions were pSP64(A)-TR $\beta$ A and pSP64(A)-RXR $\alpha$ [20] for synthesis of Xenopus TR $\beta$ and RXR $\beta$ mRNA. The capped mRNA was purified and resuspended in diethyl pyrocabonatetreated water at a concentration of $50 \mathrm{ng} / \mu \mathrm{l}$.

\section{Microinjection of Xenopus oocytes}

This was done essentially as described[20]. Briefly, plasmid $\mathrm{p} 4 / 5 \mathrm{~A}$ was injected at $23 \mathrm{nl}$ per oocyte either as ss DNA (0.12 ng/ 
oocyte) or ds DNA (0.24 ng/oocyte) into the germinal vesicle (nuclei) of the oocytes. When indicated, in vitro synthesized TR $\beta \mathrm{A}$ and $\mathrm{RXR} \alpha$ mRNAs were mixed and injected ( $27 \mathrm{nl} /$ oocyte) into the cytoplasm of oocytes $6 \mathrm{~h}$ before the DNA injection to ensure the protein expression prior to chromatin assembly. The injected oocytes were incubated at $18^{\circ} \mathrm{C}$ in the MBSH buffer[20] overnight. For transcription analysis, a group of twenty oocytes were used for each sample to minimize the variation among oocytes and injection.

\section{RNA isolation and transcription analysis}

Primer extension was used to analyze promoter activity. To isolate the RNA from the oocytes, $\mathrm{RNAzol}^{T M} \mathrm{~B}$ reagent (TELTEST, INC.) was used according to the manufacturer' $\mathrm{s}$ instruction. For primer extension, RNA from two oocytes equivalent was annealed with the end-labeled CAT1 primer (5' GGTGGTATATCCAGTGATTTTTTTCTCCAT-3' ), which hybridized to the CAT region of the transcript driven by the LTR promoter, in $10 \mu \mathrm{l}$ of $1 \mathrm{X}$ annealing buffer containing $50 \mathrm{~m}$ M Tris-HCl pH 8.3, $50 \mathrm{~m} \mathrm{M} \mathrm{KCl,} 10 \mathrm{~m} \mathrm{M} \mathrm{MgCl}_{2}, 10 \mathrm{~m}$ M DTT, 0. $5 \mathrm{mM}$ spermidine and $1 \mathrm{~m} \mathrm{M}$ of each dNTP. The annealing reaction was first preheated to $65^{\circ} \mathrm{C}$ for $10 \mathrm{~min}$, then annealed at $55^{\circ} \mathrm{C}$ for $30 \mathrm{~min}$, followed by $5 \mathrm{~min}$ incubation at $37^{\circ} \mathrm{C}$. For reverse transcription (RT), $10 \mu \mathrm{l}$ of $\mathrm{RT}$ mixture containing $2 \mathrm{X}$ annealing buffer, $5.6 \mathrm{~m} \mathrm{M}$ sodium pyrophosphate, and 1 unit of AMV reverse transcriptase (Promega) was added to the annealing mixture followed by incubation of the final mixture at $42^{\circ} \mathrm{C}$ for $1 \mathrm{~h}$. The RT products were analyzed directly on a $6 \%$ sequencing gel. For internal control, a histone $\mathrm{H} 4$ antisense primer (5'-GAGGCCGGAGATGCGCTTGAC-3' ) was included in the reaction to quantify the endogenous $\mathrm{H} 4 \mathrm{mRNA}$ level.

\section{Micrococal nuclease (MNase) digestion}

Plasmid DNA was microinjected into oocytes ( $0.5 \mathrm{ng} /$ oocyte). After overnight incubation, the oocytes were homogenized with $100 \mu$ l homogenization buffer: $10 \mathrm{~m}$ M Tris-HCl pH 8.0, $50 \mathrm{~m} \mathrm{M}$ $\mathrm{NaCl}, 1 \mathrm{~m} \mathrm{M}$ EDTA, $5 \%$ glycerol, $5 \mathrm{~m} \mathrm{M} \mathrm{MgCl}$, $3 \mathrm{~m} \mathrm{M} \mathrm{CaCl}_{2}$, and $1 \mathrm{~m}$ M DTT. Lyophilized MNase (Worthington Biochemical Corp, NJ.) was dissolved in storage buffer (50 m M Tris-HCl pH 8.0, 0 . $05 \mathrm{~m} \mathrm{M} \mathrm{C}_{\mathrm{a}} \mathrm{Cl}_{2}$, and $20 \%$ glycerol) at $100 \mathrm{U} / \mu \mathrm{l}$. Twenty oocyte equivalent of the homogenized mixture was digested with MNase at $37^{\circ} \mathrm{C}$ for $2 \mathrm{~min}$ with the enzyme concentration of $0.16 \mathrm{U}, 0.8$ $\mathrm{U}$, or $4 \mathrm{U} / 100 \mu \mathrm{l}$ reaction. The enzyme reaction was stopped with the addition of equal volume of $2 \mathrm{X}$ TNESK buffer (20 $\mathrm{m}$ M Tris$\mathrm{HCl} \mathrm{pH} \mathrm{7.4,} 200 \mathrm{~m} \mathrm{M} \mathrm{NaCl}, 2 \mathrm{~m} \mathrm{M}$ EDTA, and $2 \%$ SDS) followed by the RNAse treatment and proteinase $\mathrm{K}$ digestion. The DNA was purified by phenol-chloroform extraction and isopropanol precipitation. The purified DNA was analyzed by agarose electrophoresis and Southern hybridization.

\section{Gel mobility shift assay}

The expression and purification of the XenopusM TR $\beta$ and RXRa from E. coli was described previously[23]. To test the binding of TR $\beta /$ RXRa to the putative TRE within the HIV-1 promoter, end-labeled double-stranded oligonucleotides $(5 \times$ $10^{4} \mathrm{cpm}$ ) corresponding to the NFkB and Sp1 regions (Fig 1) were incubated with purified $\mathrm{TR} \beta / \mathrm{RXR} \alpha$ receptors for $30 \mathrm{~min}$ at room temperature in the binding buffer $\mathrm{A}$ (final $1 \mathrm{X}$ concentration: $20 \mathrm{mM}$ Tris- $\mathrm{HCl} \mathrm{pH} 7.5,5 \mathrm{mM} \mathrm{MgCl}_{2}, 100 \mathrm{~m}$ $\mathrm{M} \mathrm{KCl,} 10 \%$ glycerol, $5 \mathrm{mM}$ DTT, $0.5 \mu \mathrm{g} / \mu \mathrm{BSA}$, and $0.05 \mu \mathrm{g} /$ $\mu \mathrm{l}$ poly (dI-dC)). The reaction mixture was analyzed on a $6 \%$ polyacrylamide gel prepared with $2.6 \%$ Bis-Acrylamide and $1 /$ $2 \mathrm{X}$ TBE as the gel buffer. The gel was run at room temperature using $1 / 2 \mathrm{X}$ TBE as the running buffer. The sequence of the $\mathrm{NF} \kappa \mathrm{B}$ and Sp1 oligonucleotides were 5' AGGGACTTTCCGCTGGGGACTTTCCAGGGA-3' and 5'GGGAGGCGTGGCCTGGGCGGGACTGGGGAGTG- GC3 , respectively (sense strand). As a positive control, the ds TRE oligonucleotide of Xenopus, TR $\beta$ A gene (sense strand sequence is 5'-GATCGCAGGTCATTTCAGG ACAGC-3')[23] was used.

\section{RESULTS}

$T R / R X R$ heterodimers bind to the putative TREs in the NFkB and Sp1 sites of the HIV LTR

A number of TREs have been identified in various T3 response genes. Often, they consist of two direct repeats of AGGTCA[10-13]. The preferred binding sties for TR/RXR is two AGGTCA separated by $4 \mathrm{bp}$. Previous studies showed that both NFkB and Sp1 binding sites of the HIV LTR are capable of binding to TR/RXR[7-9], even though no easily recognizable TREs sequences are present. Thus, as a prerequisite to setting up the frog oocyte system to study LTR regulation by TR/ RXR, we first studied the binding of the putative TREs by Xenopus TR/RXR and compared it to the binding of the TRE of Xenopus TR $\beta$ A promoter, a strong TRE consisting of two near perfect repeats of AGGTCA separated by $4 \mathrm{bp}$ [23]. Gel mobility shift assay showed that oligonucleotides encompassing the NFkB or Sp1 binding sties (Fig 1) were capable of binding to Xenopus TR/RXR, although with much lower affinities than the TRE of the TR $\beta$ A promoter (Fig $2 \mathrm{~A}$, compare lanes 3 and 5 to 1).

To further compare the relative affinity, we performed gel mobility shift assay using labeled TRE of the Xenopus TR $\beta$ A promoter in the presence of unlabeled competitors. As shown in Fig 2B, as little as 20 fold excess of unlabeled TRbA TRE could efficiently competed away its own binding to TR/ RXR (compare lane 4 to 2). On the other hand, the $\mathrm{NFkB}$ oligonucleotides of HIV LTR was more than 250 fold less effective (compare lane 8, 


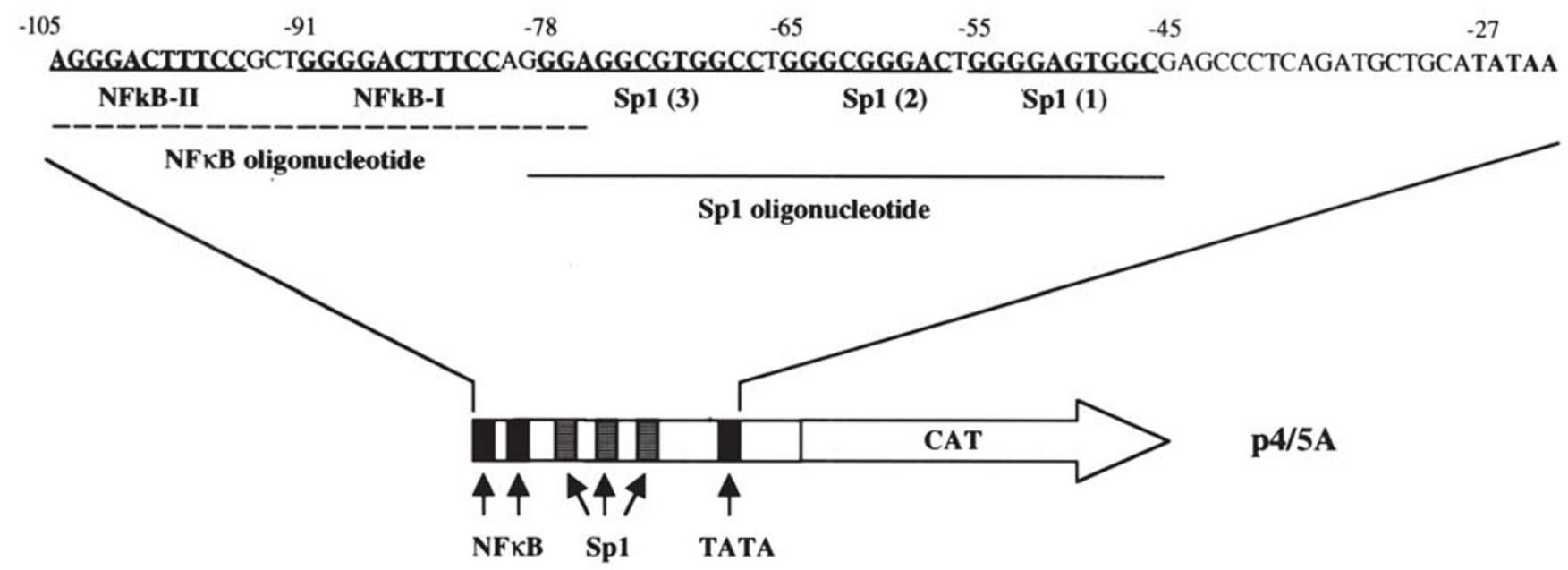

Fig 1. Schematic representation of the LTR promoter region in p4/5A. The plasmid p4/5A contained HIV-1 LTR core promoter in front of a $300 \mathrm{bp} \mathrm{CAT} \mathrm{gene} \mathrm{fragment} \mathrm{in} \mathrm{pBluescript} \mathrm{KS(-1)} \mathrm{vector.} \mathrm{The} \mathrm{promoter} \mathrm{fragment} \mathrm{included} \mathrm{two} \mathrm{NF} \kappa \mathrm{B}$ sites, three $\mathrm{Sp} 1$ sites, and TATA box. The $\mathrm{NF} \kappa \mathrm{B}$ and $\mathrm{Sp} 1$ oligonucleotides were the TRE fragments used for gel mobility shift assay.

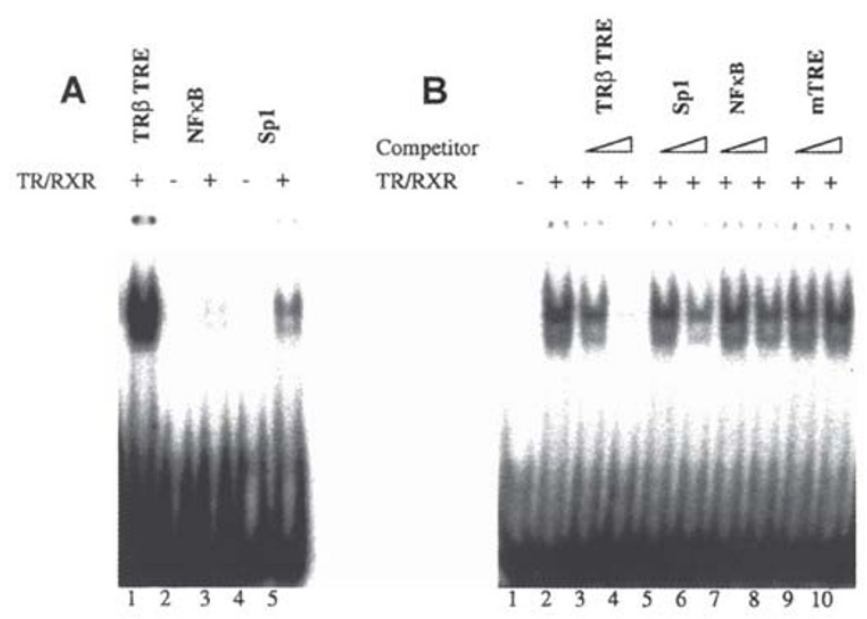

Fig 2. Gel mobility shift assay demonstrates that both the NF $\kappa$ B and Sp1 sites are weak TREs. A. TR/RXR heterodimers binds weakly to NF $\kappa$ B and $\mathrm{Sp} 1$ sites in the LTR. E. coli, produced, purified TR and RXR was incubated with ${ }^{32} \mathrm{P}$-labeled ds oligonucleotides corresponding to the TRE of Xenopus TR $\beta$ A gene (TR $\beta$ TRE)[23], the $\mathrm{NF} \kappa \mathrm{B}$, or Sp1 sites (Fig 1) and the resulting complexes were analyzed on a native polyacrylamid gel. B. Competition assay demonstrates that the $\mathrm{Sp} 1$ and $\mathrm{NF} \kappa \mathrm{B}$ sites in HIV LTR are weak TREs. Purified TR and RXR was incubated with32P-labeled TR $\beta$ TRE alone or in the presence of unlabeled TR $\beta$ TRE (2x in lane 3 or 20x in lane 4), Sp1 sites of LTR (50x in lane 5 or $500 \mathrm{x}$ in lane 6 ), NFkB sites of LTR (50x in lane 7 or $500 \mathrm{x}$ in lane 8), or mutated, non functional TRE of TRbA gene (mTRE) (50x in lane 9 or 500x in lane 10). Note that TR $\beta$ TRE competed effectively, $\mathrm{Sp} 1$ and $\mathrm{NF} \kappa$ B competed weakly, while mTRE did not compete. where a 500 fold of $\mathrm{NF} \kappa \mathrm{B}$ oligonucleotide were present, to lane 3 , where only 2 fold of TR $\beta$ A TRE were present). The Sp1 oligonucleotide of HIV LTR was about 2 fold more effective than the $\mathrm{NF} \kappa \mathrm{B}$ oligonucleotide, in agreement with the binding data in Fig 2A. Under the same conditions, a mutant TRbA TRE (mTRE) showed no competition at all (Fig 2B, lanes 9 and 10), supporting that the HIV LTR oligonucleotides were TREs, although weak ones.

Unliganded TR/RXR heterodimer represses $L T R$ in chromatin in vivo

To investigate the role of TR in transcriptional regulation of HIV LTR under conditions that mimic those in somatic cells, we took advantage of the ability of the frog oocyte to replicate and concurrently chromatinize exogenous ss plasmid DNA injected into its nucleus, similar to that takes place in somatic cells[19]. Thus, we replaced the core promoter sequence, containing the TATA box, binding sites for $\mathrm{NF} \kappa \mathrm{B}$ and $\mathrm{Sp} 1$, in front of a $\mathrm{CAT}$ reporter in a plasmid vector and microinjected the resulting plasmid (p4/5A) into the nucleus of Xenopus oocyte in either the ss or ds form. After overnight incubation of the oocytes, the plasmid minichromosome was isolated and treated with micrococcal nuclease (MNase), which digested the internucleoleosomal regions. Southern blot analysis of the digested prod- 


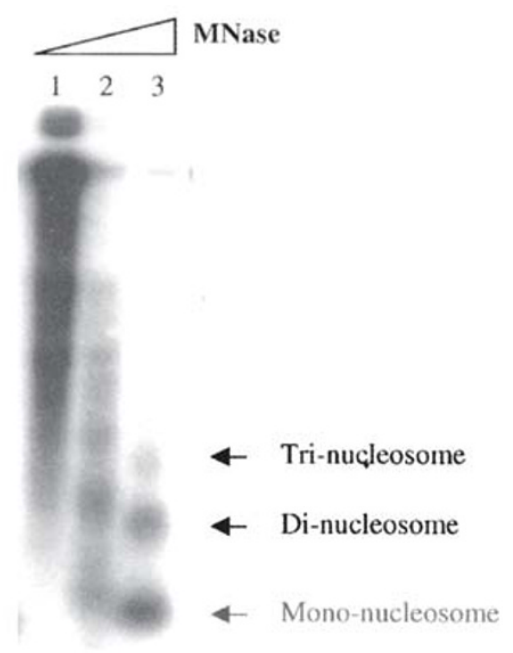

Fig 3. LTR is chromatinized in the oocyte nucleus. The ss $\mathrm{p} 4 / 5 \mathrm{~A}$ plasmid was microinjected into oocyte nucleus. After overnight incubation, the plasmid minichromosome was isolated and digested with different concentrations of micrococcal nuclease (MNase). The DNA was then isolated and subjected to Southern blot hybridization with a ${ }^{32} \mathrm{P}-$ labeled probe corresponding to the LTR. Note the presence of a nucleosomal ladder, indicative of the presence of an ordered nucleosome array at the LTR and flanking sequences. The same pattern was obtained when the same blot was reprobed with a probe made from the entire plasmid (not shown), suggesting the existence of uniformly distributed nucleosomes on the plasmid.

ucts showed that the plasmid was found to be chromatinized as reflected by the presence of the nucleosomal sized DNA ladder (Fig 3). Although this assay can detect some difference in chromatin formed by certain ss vs. ds plasmid DNAs[16], under the conditions used, both ds and ss $\mathrm{p} 4 / 5 \mathrm{~A}$ plasmid DNAs were found to produce similar digestion patterns (Fig 3 and data not shown), suggesting that the differences, if any, between the two types of chromatin formed with ss and ds DNA, were too subtle to be detected by this assay.

To establish a $\mathrm{T}_{3}$-dependent oocyte system, we injected mRNAs encoding Xenopus TR $\beta$ and RXRa into the cytoplasm of the oocytes to allow the synthesis and nuclear accumulation of TR and RXR [20]. Six h later, we injected ds and ss p4/5A plasmid into the oocyte nuclei. Following overnight incubation, RNA was isolated from the oocytes and subjected to primer extension assays to determine HIV LTR promoter activity. The results showed that the LTR had similarly activity when either the ds or ss p4/5A DNA was used (Fig 4 compare lanes 1 and 5), in contrast with the observations for some other promoters[16], consistent with the idea that chromatin dependent repression was promoterdependent.

The addition of $\mathrm{T}_{3}$ alone had little effect on LTR activity regardless of the form of the plasmid DNA used (Fig 4). On the other hand, over expression of $\mathrm{TR} / \mathrm{RXR}$ in the absence of $\mathrm{T}_{3}$ caused the repression of the promoter and the addition of $\mathrm{T}_{3}$ relieved the repression and further activated the LTR (Fig 4, compare lane 3 to 1 or 4 for ds DNA and lane 7 to 5 or 8 for ss DNA). In contrast to the lack of any effect of chromatin on LTR activity in the absence of TR/RXR, replication-coupled chromatin, i.e., when ss DNA was used, enhanced the repression by unliganded TR/RXR, leading to a lower level of LTR activity (Fig 4, compared lane 7 to 3 ). Unliganded TR/RXR caused a 3.5 fold or 7.5 fold repression when ds or ss LTR plasmid DNA was used, respectively, and overall activation by $\mathrm{T}_{3}$ was 5.5 fold for ds $\mathrm{p} 4 / 5 \mathrm{~A}$ but 12 fold for ss $\mathrm{p} 4 / 5 \mathrm{~A}$. Thus, maximal regulation by $\mathrm{T}_{3}$ was observed under conditions closely mimicking those in somatic cells, $i$. e., when TR/RXR was present during replicationcoupled chromatin assembly.

Inhibition of histone deacetylases reverses repression by unliganded TR/RXR

Studies in a variety of systems show that to regulate transcription, TR/RXR recruits to its target promoters coactivators or corepressors in the presence or absence of $\mathrm{T}_{3}$, leading to transcriptional activation or repression, respectively. Interestingly, many coactivators such SRC-1 and CBP/p300 are histone acetylases (acetyltransferases) while corepressors such as SMRT and N-CoR can associate with histone deacetylase such as RPD3[24-27]. To test the effect of histone acetylation on TR-dependent HIV LTR activity, we microinjected TR/RXR mRNAs and $\mathrm{p} 4 / 5 \mathrm{~A}$ into frog oocytes and treated the oocytes with trichostatin A (TSA), a specific histone deacetylase inhibitor[21]. The treatment led to the relief of the transcriptional repression caused by unliganded TR/RXR when either ss or ds p4/5A was used (Fig 5). The effect of TSA was essentially identical to $\mathrm{T}_{3}$, although TSA alone was able to 


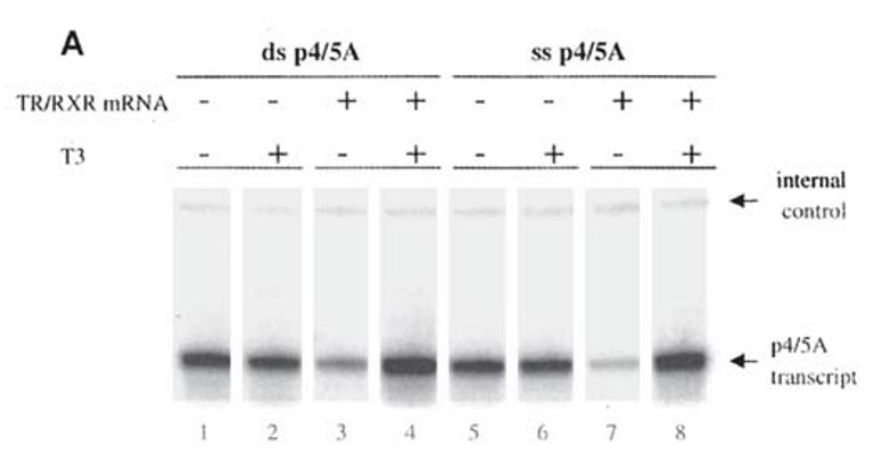

B

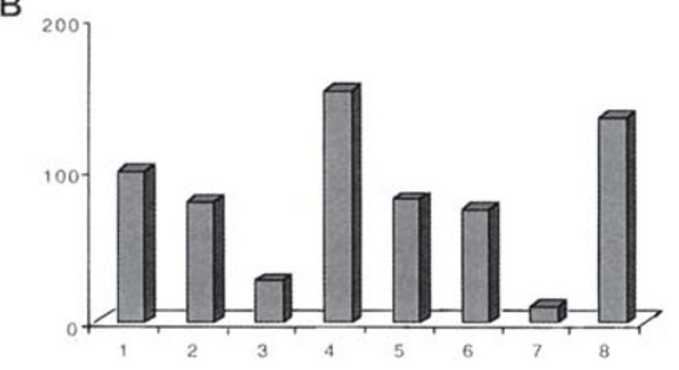

Relative

intensity

$\begin{array}{llllllll}100 & 80 & 28 & 153 & 82 & 75 & 11 & 135\end{array}$

Fig 4. Replication-coupled chromatin assembly synergizes the repression of LTR by unliganded TR $\beta / \mathbf{R X R} \alpha$ in oocytes. A. Oocytes were injected with (+) or without (-) TR $\beta / R X R \alpha$ mRNAs 6 h before the injection of ss or ds p4/5A plasmid containing the HIV LTR promoter. The injected oocytes were treated overnight with $50 \mathrm{n} \mathrm{M} \mathrm{T}$ as indicated and the promoter activity was analyzed by primer extension using CAT1 primer on the RNA isolated from the oocytes. A histone $\mathrm{H} 4$ gene antisense primer was included as a control in the primer extension reaction to quantitate the endogenous H4 mRNA level. B. PhosphorImager quantification of the data in (A), normalized against the endogenous $\mathrm{H} 4$ signal. Note that in the absence of TR, both ds and ss p4/5A showed significant promoter activity (Lanes 1 and 5 with activity in lane 1 arbitrarily set to 100) and the addition of $\mathrm{T}_{3}$ had little effect (Lanes 2 and 6). The presence of TR/RXR reduced the promoter activity more effectively when ss $\mathrm{p} 4 / 5 \mathrm{~A}$ was used (3.6-fold for ds p $4 / 5 \mathrm{~A}$ and 7.5-fold for ss p4/5A; Lanes 3 and 7). The addition of $\mathrm{T}_{3}$ reversed this inhibition and led to further activation (5.5fold induction by $\mathrm{T}_{3}$ for ds $\mathrm{p} 4 / 5 \mathrm{~A}$ and 12 -fold for ss $\mathrm{p} 4 / 5 \mathrm{~A}$; Lanes 4 and 8).

activate the LTR slightly (Fig 5, compare lane 2 to 1 and 7 to 6 ). These results suggest that TR/RXR recruits deacetylases through corepressors in the absence of $\mathrm{T}_{3}$ to repress the transcription of HIV LTR while recruiting coactivators with acetylase activities in the presence of $T_{3}$ to activate the LTR.

\section{DISCUSSION}

The HIV LTR is known to be regulated by a
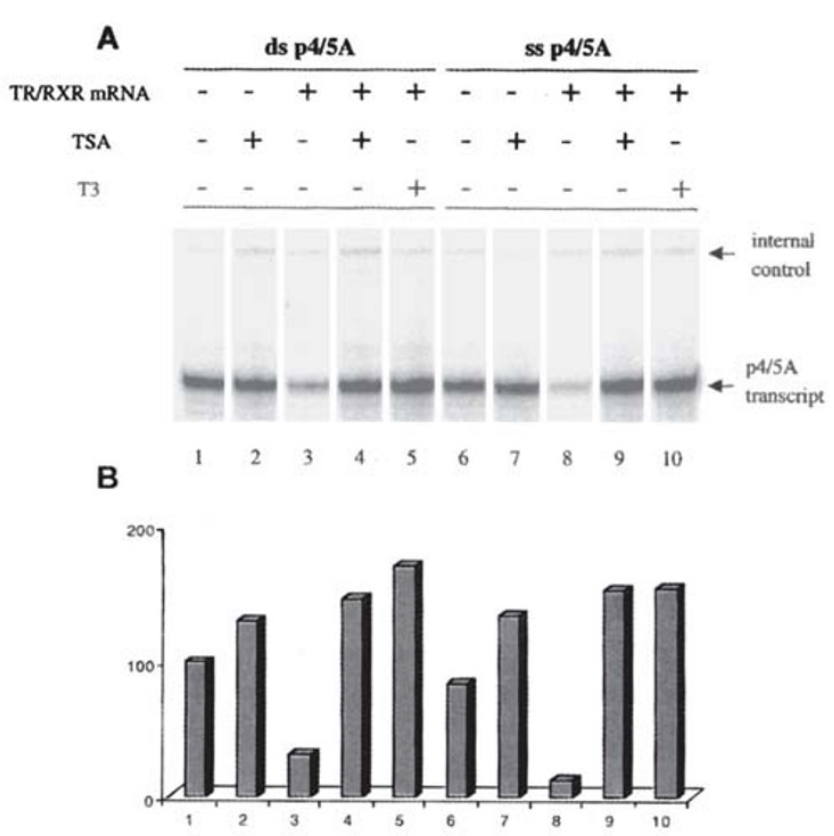

$\begin{array}{lllllllllll}\begin{array}{l}\text { Relative } \\ \text { intensity }\end{array} & 100 & 130 & 31 & 147 & 171 & 85 & 134 & 12 & 153 & 155\end{array}$

Fig 5.Participation of histone deacetylases in transcription regulation of LTR by TR. A. Oocytes were injected as in Fig 4. The injected oocytes were treated with or without $5 \mathrm{ng} / \mathrm{ml} \mathrm{TSA}$ or $50 \mathrm{n} \mathrm{M} \mathrm{T}_{3}$ as indicated and the promoter activity was analyzed by primer extension. Again, the histone $\mathrm{H} 4$ antisense primer was included in the primer extension reaction as a control. B. PhosphorImager quantification of the data in (A). In the absence of TR, both ds and ss p4/5A showed significant promoter activity (Lanes 1 and 6 with the activity in lane 1 set to 100). The addition of TSA activated the promoter slightly (Lanes 2 and 7). The presence of unliganded TR/RXR repressed the promoter activity, again more dramatically on ss DNA (3.2-fold for ds p4/5A and 7.1-fold for ss p4/5A; Lanes 3 and 8). The addition of TSA reversed the inhibition (4.7-fold induction by TSA for ds p4/5A and 13 -fold for ss $\mathrm{p} 4 / 5 \mathrm{~A}$; Lanes 4 and 9). The addition of $\mathrm{T}_{3}$ had essentially the same effects (5.5-fold induction by $\mathrm{T}_{3}$ for $\mathrm{ds} \mathrm{p} 4 / 5 \mathrm{~A}$ and 13 fold for ss $\mathrm{p} 4 / 5 \mathrm{~A}$; Lanes 5 and 10), supporting a role of histone deacetylase in the repression by unliganded TR/RXR.

variety of cellular factors. The role of TR in LTR regulation has been controversial. Our studies here demonstrate that under chromatin conditions mimicking the natural environment where the LTR is active, TR/RXR heterodimer activates the LTR in the presence of $\mathrm{T}_{3}$, confirming the findings by two groups[7,9] but disagreeing with those by the third[8]. At least two factors may contribute to the different results of Rahman et 
al[8]. These are the lack of proper chromatin organization and the choice of the cell line used for the transfection study. On the other hand, all studies demonstrate that TR/RXR can bind to both $\mathrm{NFkB}$ and $\mathrm{Sp} 1$ sites in the proximal promoter region (Fig 1), arguing the in vivo, TR/RXR are likely to affect LTR activity. More importantly, our studies here demonstrate for the first time that in the absence of $\mathrm{T}_{3}$, TR/RXR dramatically represses HIV LTR and that replication-coupled chromatin assembly synergizes with unliganded TR/RXR to effectively repress LTR. Another important conclusion from our studies here is that histone deacetylase plays a critical role in transcriptional regulation of LTR, especially the repression by unliganded TR/RXR.

The integration of HIV viral genome into host cell chromosome ensures that the viral genome is chromatinized in vivo. However, the presence of multiple binding sites for several abundant, ubiquitous transcription factors such as $\mathrm{NF}_{\kappa} \mathrm{B}$ and $\mathrm{Sp} 1$ in the LTR may suggest that the LTR is bound by transcription factors and thus free of chromatin. Our data demonstrate that the promoter region is chromatinized with an ordered nucleosomal array. On the other hand, transcription factors and transcriptional machinery can still access the promoter in the chromatin context as reflected by the high levels of basal activity under both chromatinization conditions. Furthermore, chromatin assembly facilitates the regulation by $\mathrm{TR} /$ RXR, suggesting that the virus may make use of host cell chromatin assembly machinery for proper regulation of the LTR during its passage through host cells.

Numerous studies have shown that chromatin can inhibit promoter activity. In the frog oocyte, replication-coupled chromatin assembly represses the basal activity of a number of promoters, including $\mathrm{T}_{3}$-inducible promoter of the Xenopus $\mathrm{TRbA}$ gene[16]. Interestingly, this process has little effect on HIV LTR as the basal LTR activity was found to be similar regardless of use of ds or ss plasmid. This may not be surprising as the core promoter of LTR contains not only a TATA box but also several binding sites for abundant, ubiquitous transcription factors $\mathrm{Sp} 1$ and $\mathrm{NF}_{\kappa} \mathrm{B}$. It is likely that these factors compete effectively for DNA binding with chromatin such that they are bound to the target sites even when the LTR is chromatinized. Thus, the promoter remains active regardless of chromatin assembly pathways. On the other hand, chromatin assembly facilitates repression by unliganded TR/RXR, similar to what we observed earlier on the $\mathrm{T}_{3}$-inducible Xenopus TRbA promoter[16]. The mechanism underlying the synergism is unclear but it is possible that the binding of TR/RXR facilitates the assembly of a more repressed chromatin state.

It is clear from our studies here as well as those reported earlier that $\mathrm{TR} / \mathrm{RXR}$ binds to sites overlapping with $\mathrm{Sp} 1$ and $\mathrm{NF} \kappa \mathrm{B}$ recognition sequences [7-9]. It has been shown that in vitro TR/RXR and $\mathrm{Sp} 1$ or $\mathrm{NF} \kappa \mathrm{B}$ binding is mutually exclusive [7]. On the other hand, given the existence of multiple sites for each of these factors in the LTR, if is quite possible that all these factors can reside on the LTR simultaneously with TR/RXR and NF $\kappa$ B or Sp1 occupying different sites. Our earlier studies have shown that the binding of TR/ RXR heterodimer to a single site is as effective as multiple TR/RXR heterodimers bound to several consecutive sites at a single location[17]. Thus, simultaneous binding of TR/RXR, Sp1, and NF $k$ $\mathrm{B}$ to LTR is not only physically possible but also allows TR/RXR to effectively regulate the promoter in the presence of other cellular factors within a chromatin context.

The exact mechanisms by which TR/RXR regulates HIV LTR is unclear. It is generally believed that unliganded TR/RXR represses transcription by recruiting corepressor complexes while T3bound TR/RXR activates transcription by recruiting coactivator complexes. The corepressor complexes contain histone deacetylases while some of the coactivator complexes have histone acetyltransferases activity but others do not[2428]. This has led to the proposal of multiple models. Regardless of the exact model, in vivo, it is likely that chromatin will influence this process. Indeed, our system, mimicking HIV in somatic cells, clearly reveals the importance of replicationcoupled chromatin assembly in LTR repression by unliganded TR/RXR. More importantly, our studies using a specific histone deacetylase inhibitor show that unliganded TR/RXR utilizes chromatin to effectively repress HIV LTR by employing histone deacetylase(s). In addition, we also 
observed a slight activation of the basal activity of the LTR with TSA alone (in the absence of TR/ RXR), sugges- ting deacetylated or less acetylated chromatin is inhibitory to LTR; in agreement with the in vitro transcription study reported earlier[29].

In conclusion, we have shown here that under conditions that reflects the in vivo environment of HIV in somatic cells, unliganded TR/RXR synergizes with chromatin assembly to repress HIV LTR. $\mathrm{T}_{3}$ binding to TR relieves the repression and leads to further activation of the promoter above the basal level. We further demonstrate that histone acetylation plays an important role in transcriptional regulation by TR/RXR. Given the known association of $\mathrm{T}_{3}$ levels with various HIV pathological conditions, our studies suggest that it may be possible to effectively inhibit HIV propagation by simultaneously control TR/RXR activity and histone acetylation levels.

\section{ACKNOWLEDGEMENTS}

We would like to thank Dr. T. Collingwood for the gift of over expressed TR and RXR and Ms. K. Pham for preparing the manuscript.

\section{REFERENCES}

[1] Pereira LA, Bentley K, Peeters A, Churchill MJ, Deacon NJ. A compilation of cellular transcription factor interactions with the HIV-1 LTR promoter. Nuclei Acids Research 2000; 28(3):663-8.

[2] Vaishnav NW, Wong-Staal F. The biochemistry of AIDS. Annu Rev Biochem 1991; 60:577-630.

[3] Lopresti JS, Fried JC, Spencer CA, Nicoloff JT. Uniquie Alterations of Thyroid-Hormone indexes in the acquired immunodeficiency syndrome (Aids). Annals of Internal Medicine 1989; 110(12):970-5.

[4] Tang WW, Kaptein EM. Thyroid-Hormone Levels in the Acquired immunodeficiency syndrome (Aids). Western Journal of Medicine 1980; 151(6):627-31.

[5] Bernal J, Andersson LC. The nuclear 3, 5, 3' -triiodothyronine receptor in human leukaemic cell lines. Acta Endocrinol 1984; 105:429-32

[6] Oppenheimer JH, Schwartz HL, Mariash CN, Kinlaw WB, Wong NCW, Freake HC. Advances in our understanding of thyroid hormone action at the cellular level. Endocr Rev 1987; 8:288-308.

[7] Desai-Yajnik V, Samuels H. The NF- $\kappa$ B and Sp1 Motifsof the Human Immunodeficiency Virus Type 1 Long Terminal Repeat Function as Novel Thyroid Hormone Response Elements. Molecular and Cellular Biol. 1993; 13 (8):5057-69.

[8] Rahman A, Esmaili A, Saatcioglu F. A Unique Thyroid
Hormone Response Element in the Human Immunodeficiency Virus Type 1 Long Terminal Repeat That Overlaps the Sp1 Binding Sites. J Biol Chem 1995; 270(52): 31059-64.

[9] Xu, J, Luznik L, Wong-Staal F, Gill GN. Hormone Receptor Regulation of the Human Immunodeficiency Virus Type 1 and type 2 Long Terminal Repeats. J Biomed Sci 1996; 3:323-31.

[10] Tsai M-J, O'Malley BW. Molecular mechanisms of action of steroid/thyroid receptor superfamily members. Ann Rev Biochem 1994; 63:451-86.

[11] Shi YB, Wong J, Puzianowska-Kuznicka M. Thyroid hormone receptors: Mechanisms of transcriptional regulation and roles during frog development. J Biomed Sci 1996; 3:307-18.

[12] Mangelsdorf DJ, Thummel C, Beato M, Herrlich P, Schutz G, Umesono K, Blumberg B, Kastner P, Mark M, Chambon P, Evans RM. The nuclear receptor superfamily: The second decade. Cell 1995; 83:835-39.

[13] Yen PM, Chin WW. New advances in understanding the molecular mechanisms of thyroid hormone action. Trends Endocrinol.Metab. 1994; 5:65-72.

[14] Puzianowska-Kuznicka M, Wong J, Kanamori A, Shi YB. Functional characterization of a mutant thyroid hormone receptor in Xenopus laevis. J Biol Chem 1996; 271: 33394-403.

[15] Puzianowska-Kuznicka M, Damjanovski S, Shi Y-B. Both thyroid Hormone and 9-cis Retinoic Acid Receptors Are Required To Efficiently mediate the Effects of Thyroid Hormone on Embryonic Development and Specific Gene Regulation in Xenopus laevis. Mol And Cell Biol 1997; 17:4738-49.

[16] Wong J, Shi Y-B, Wolffe AP. A role for nucleosome assembly in both silencing and activation of the Xenopus TrbA gene by the thyroid hormone receptor. Genes and Development 1995; 9:2696-711.

[17] Wong J, Shi Y-B, Wolffe A P. Determinants of chromatin disruption and transcriptional regulation instigated by the thyroid hormone receptor: Hormone regulated chromatin disruption is not sufficient transcriptional activation. EMBO J 1997; 16:3158-71.

[18] Woffe AP. Chromatin remodeling regulated by steroid and nuclear receptors. Cell Research 1997; 7:127-42.

[19] Almouzni G, Clark DJ, Mechali M, Wolffe AP. Chromatin assembly on replicating DNA in vitroM. Nucl Acids Res 1990; 18:5767-74.

[20] Wong J, Shi Y-B. Coordinated regulation of and transcriptional activation by Xenopus thyroid hormone and retinoid X receptors. J Biol Chem 1995; 270:18479-83.

[21] Yoshida M, Horinouchi S, Beppu T. Trichostatin A and trapoxin: novel chemical probes for the role of histone acetylation in chromatin structure and function. BioEssays 1995; 17:423-30.

[22] Maniatis T, Fritsch EF, Sambrook J. Molecular cloning: A laboratory manual. Cold Spring Harbor Laboratory Press, Cold Spring Harbor, New York 1982.

[23] Ranjan M, Wong J, Shi Y-B. Transcriptional repression of Xenopus $\mathrm{TRb}$ gene is mediated by a thyroid hormone response element located near the start site. Biol Chem 1994; 269:24699-705. 
[24] Koenig RJ. Thyroid hormone receptor coactivators and corepressors. Thyroid 1998; 8:703-13.

[25] Chen JD, Li H. Coactivation and corepresion in transcriptional regulation by steroid/nuclear hormone receptors. Critical Reviews in Eukaryotic Gene Express 1998; 8:169-90.

[26] McKenna NJ, Lanz RB, O'Malley BW. Nuclear Receptor Coregulators: Cellular and Molecular Biology. Endocrine Reviews 1999; 20:321-44.

[27] Xu L, Glass CK, Rosenfeld MG. Coactivator and core- pressor complexes in nuclear receptor function. Current Opinion in Genetics and Development 1997; 9:140-7.

[28] Rachez C, Freedman LP. Mechanisms of gene regulation by vitamin D3 receptor: a network of coactivator interactions. Gene 2000; 246:9-21.

[29] Sheridan PL, Mayall TP, Verdin E, Jones KA. Histone acetyltransferases regulate HIV-1 enhancer activity in vitro. Genes and Dev 1997; 11:3327-40. 